\title{
On the striction curves along the involutive and Bertrandian Darboux ruled surfaces based on the tangent vector fields
}

\author{
Seyda Kilicoglu ${ }^{1}$, Suleyman Senyurt ${ }^{2}$ and Abdussamet Caliskan ${ }^{3}$ \\ ${ }^{1}$ Faculty of Education, Department of Mathematics, Baskent University, Ankara, Turkey \\ ${ }^{2,3}$ Faculty of Arts and Sciences, Department of Mathematics, Ordu University, Ordu, Turkey \\ Received: 18 February 2016, Accepted: 26 Semptember 2016 \\ Published online: 29 October 2016.
}

\begin{abstract}
In this paper, we consider special ruled surfaces associated to the involute curve $\alpha^{*}$ and Bertrand mate $\alpha^{* *}$ of a curve $\alpha$. They are called as Involutive Darboux Frenet ruled surface and Bertrandian Darboux Frenet ruled surface, cause of their generators are the Darboux vector fields of involute curve $\alpha^{*}$ and Bertrand mate $\alpha^{* *}$. We give the parametric equations of striction curves along these Frenet ruled surfaces in terms of the Frenet apparatus of curve $\alpha$. Further some results are given based on the tangent vector fields of these striction curves along Involutive and Bertrand Darboux ruled surfaces in $\mathrm{E}^{3}$.
\end{abstract}

Keywords: Involute curve, Bertrand curves pair,striction curves, Bertrandian Darboux ruled surfaces, involutive Darboux ruled surfaces.

\section{Introduction and preliminaries}

The Euclidean 3-space $E^{3}$ be inner product given by

$$
\langle,\rangle=x_{1}^{2}+x_{2}^{3}+x_{3}^{2}
$$

where $\left(x_{1}, x_{2}, x_{3}\right) \in E^{3}$. Let $\alpha: I \rightarrow E^{3}$ be a unit speed curve denote by $\left\{V_{1}, V_{1}, V_{1}\right\}$ the moving Frenet frame . For an arbitrary curve $\alpha \in E^{3}$, with first and second curvature, $k_{1}$ and $k_{2}$ respectively, the Frenet formulae is given by [2]

$$
\left\{\begin{array}{l}
V_{1}^{\prime}=k_{1} V_{2} \\
V_{2}^{\prime}=-k_{1} V_{1}+k_{2} V_{3} \\
V_{3}^{\prime}=-k_{2} V_{2} .
\end{array}\right.
$$

For any unit speed curve $\alpha: I \rightarrow \mathbb{E}^{3}$, the vector $D$ is called Darboux vector defined by $D=k_{2} V_{1}+k_{1} V_{3}$. Let a vector field be along $\alpha(s)$

$$
\tilde{D}(s)=\frac{k_{2}}{k_{1}}(s) V_{1}(s)+V_{3}(s)
$$

under the condition that $k_{1}(s) \neq 0$ and it is called the modified Darboux vector field of $\alpha$.[4].

Deriving curves based on the other curves is a subject in geometry. Involute-evolute curves, Bertrand curves pair are this kind of curves. Let unit speed regular curve $\alpha: I \rightarrow \mathbb{E}^{3}$ and $\alpha^{*}: I \rightarrow \mathbb{E}^{3}$ be given. For $\forall s \in I$, then the curve $\alpha^{*}$ is called the involute of the curve $\alpha$, if the tangent at the point $\alpha(s)$ to the curve $\alpha$ passes through the tangent at the point $\alpha^{*}(s)$ to 
the curve $\alpha^{*}$, then we can write

$$
\alpha^{*}(s)=\alpha(s)+(c-s) V_{1}(s), c=\text { const }
$$

The distance between corresponding points of the involute curve in $\mathbb{E}^{3}$ is $d\left(\alpha(s), \alpha^{*}(s)\right)=|c-s|, c$ is constant,$\forall s \in I$, ([2],[5]).

Theorem 1.The Frenet vector fields of the involute $\alpha^{*}$, based on the its evolute curve $\alpha$ are

$$
\left\{\begin{array}{l}
V_{1}^{*}=V_{2} \\
V_{2}^{*}=\frac{-k_{1}}{\left(k_{1}^{2}+k_{2}^{2}\right)^{\frac{1}{2}}} V_{1}+\frac{k_{2}}{\left(k_{1}^{2}+k_{2}^{2}\right)^{\frac{1}{2}}} V_{3} \\
V_{3}^{*}=\frac{k_{2}}{\left(k_{1}^{2}+k_{2}^{2}\right)^{\frac{1}{2}}} V_{1}+\frac{k_{1}}{\left(k_{1}^{2}+k_{2}^{2}\right)^{\frac{1}{2}}} V_{3}
\end{array}\right.
$$

and

$$
\tilde{D}^{*}=\frac{k_{2}}{\left(k_{1}^{2}+k_{2}^{2}\right)^{\frac{1}{2}}} V_{1}-\frac{k_{1}^{\prime} k_{2}-k_{1} k_{2}^{\prime}}{\left(k_{1}^{2}+k_{2}^{2}\right)^{\frac{3}{2}}} V_{2}+\frac{k_{1} V_{3}}{\left(k_{1}^{2}+k_{2}^{2}\right)^{\frac{1}{2}}}
$$

The first curvature and second curvature of involute $\alpha^{*}$ are, respectively

$$
k_{1}^{*}=\frac{\sqrt{k_{1}^{2}+k_{2}^{2}}}{(c-s) k_{1}}, k_{2}^{*}=\frac{-k_{2}^{2}\left(\frac{k_{1}}{k_{2}}\right)^{\prime}}{(c-s) k_{1}\left(k_{1}^{2}+k_{2}^{2}\right)},[5] .
$$

Let $\alpha: I \rightarrow \mathbb{E}^{3}$ and $\alpha^{* *}: I \rightarrow \mathbb{E}^{3}$ be the $C^{2}$ - class differentiable unit speed two curves and let $V_{1}(s), V_{2}(s), V_{3}(s)$ and $V_{1}^{* *}(s), V_{2}^{* *}(s), V_{3}^{* *}(s)$ be the Frenet frames of the curves $\alpha$ and $\alpha^{* *}$, respectively. If the principal normal vector $V_{2}$ of the curve $\alpha$ is linearly dependent on the principal normal vector $V_{2}^{* *}$ of the curve $\alpha^{* *}$, then the pair $\left\{\alpha, \alpha^{* *}\right\}$ are called Bertrand curves pair. Also $\alpha^{* *}$ is called Bertrand mate of $\alpha$. If the curve $\alpha^{* *}$ is Bertrand mate of $\alpha$, then we may write that

$$
\alpha^{* *}(s)=\alpha(s)+\lambda V_{2}(s)
$$

The measure of the angle between the vector fields of Bertrand curves pair and distance between corresponding points of the Bertrand curves pair are constant. If $k_{2}(s) \neq 0$ along $\alpha(s)$, then $\alpha(s)$ is a Bertrand curve if and only if there exist nonzero real numbers $\lambda$ and $\beta$ such that constant $\lambda k_{1}+\beta k_{2}=1$ for any $s \in I$. It follows from this fact that a circular helix is a Bertrand curve, [2],[5].

Theorem 2.Let $\alpha: I \rightarrow \mathbb{E}^{3}$ and $\alpha^{* *}: I \rightarrow \mathbb{E}^{3}$ be the $C^{2}$ - class differentiable unit speed two curves and the quantities $\left\{V_{1}, V_{2}, V_{3}, \tilde{D}, k_{1}, k_{2}\right\}$ and $\left\{V_{1}^{* *}, V_{2}^{* *}, V_{3}^{* *}, \tilde{D}^{* *}, k_{1}^{* *}, k_{2}^{* *}\right\}$ are collectively Frenet-Serret apparatus and modified Darboux vector of the curves $\alpha$ and the Bertrand mate $\alpha^{* *}$, respectively, then

$$
\left\{\begin{array}{l}
V_{1}^{* *}=\frac{\beta}{\sqrt{\lambda^{2}+\beta^{2}}} V_{1}+\frac{\lambda}{\sqrt{\lambda^{2}+\beta^{2}}} V_{3} \\
V_{2}^{* *}=V_{2} \\
V_{3}^{* *}=\frac{-\lambda}{\sqrt{\lambda^{2}+\beta^{2}}} V_{1}+\frac{\beta}{\sqrt{\lambda^{2}+\beta^{2}}} V_{3} ; \lambda k_{2}>0
\end{array}\right.
$$

and

$$
\tilde{D}^{* *}=\frac{\sqrt{\lambda^{2}+\beta^{2}}}{\left(\beta k_{1}-\lambda k_{2}\right)}\left(k_{2} V_{1}+k_{1} V_{3}\right)
$$


The first and second curvatures of the offset curve $\alpha^{* *}$ are given by

$$
\left\{\begin{array}{l}
k_{1}^{* *}=\frac{\beta k_{1}-\lambda k_{2}}{\left(\lambda^{2}+\beta^{2}\right) k_{2}}=\frac{k_{1}-\lambda\left(k_{1}^{2}+k_{2}^{2}\right)}{\left(\lambda^{2}+\beta^{2}\right) k_{2}^{2}} \\
k_{2}^{* *}=\frac{1}{\left(\lambda^{2}+\beta^{2}\right) k_{2}},[6]
\end{array}\right.
$$

Due to this theorem, we can write

$$
\left(\frac{k_{2}^{* *}}{k_{1}^{* *}}\right)^{\prime}=\left(\frac{-m^{\prime}}{m^{2}}\right) \frac{1}{k_{2} \sqrt{\lambda^{2}+\beta^{2}}},
$$

where $\beta k_{1}-\lambda k_{2}=m$ and $\frac{d s}{d s^{* *}}=\frac{1}{k_{2} \sqrt{\lambda^{2}+\beta^{2}}}$.

A ruled surface in $\mathbb{E}^{3}$ is a surface which contains at least one 1-parameter family of straight lines. Thus a ruled surface has a parametrization

$$
\varphi(s, v)=\alpha(s)+v x(s)
$$

where $\alpha$ and $x$ are curves in $\mathbb{E}^{3}$. We call $\varphi$ a ruled patch. The curve $\alpha$ is called the directrix or base curve of the ruled surface, and $x$ is called the director curve, [1]. The striction point on a ruled surface is the foot of the common normal between two consecutive generators (or ruling). The set of striction points defines the striction curve given by

$$
c(s)=\alpha(s)-\frac{\left\langle\alpha_{s}, x_{s}\right\rangle}{\left\langle x_{s}, x_{s}\right\rangle} x(s),[1] .
$$

Let $\alpha^{*}(s)$ be involute of $\alpha(s)$ curve. It is taken Frenet vectors of involut curve instead of generator vectors in equation (12), the equaitons

$$
\left\{\begin{array}{l}
\varphi_{1}^{*}\left(s, v_{1}\right)=\alpha(s)+(c-s) V_{1}(s)+v_{1} V_{2}(s), \\
\varphi_{2}^{*}\left(s, v_{2}\right)=\alpha(s)+(c-s) V_{1}(s)+v_{2}\left(\frac{-k_{1} V_{1}+k_{2} V_{3}}{\left(k_{1}^{2}+k_{2}^{2}\right)^{\frac{1}{2}}}\right), \\
\varphi_{3}^{*}\left(s, v_{3}\right)=\alpha(s)+(c-s) V_{1}(s)+v_{3}\left(\frac{k_{2} V_{1}+k_{1} V_{3}}{\left(k_{1}^{2}+k_{2}^{2}\right)^{\frac{1}{2}}}\right),
\end{array}\right.
$$

are the parametrization of the ruled surfaces which are called involutive tangent ruled surface, involutive normal ruled surface and involutive binormal ruled surface, respectively [3]. Let $\left(\alpha, \alpha^{* *}\right)$ be Bertrand curves pair. It is taken Frenet vectors of Bertrand mate curve instead of generator vectors in equation (12), the equaitons

$$
\left\{\begin{array}{l}
\varphi_{1}^{* *}\left(s, w_{1}\right)=\alpha+\lambda V_{2}+w_{1} \frac{\beta V_{1}+\lambda V_{3}}{\sqrt{\lambda^{2}+\beta^{2}}} \\
\varphi_{2}^{* *}\left(s, w_{2}\right)=\alpha+\left(\lambda+w_{2}\right) V_{2} \\
\varphi_{3}^{* *}\left(s, w_{3}\right)=\alpha+\lambda V_{2}+w_{3}\left(\frac{-\lambda V_{1}+\beta V_{3}}{\sqrt{\lambda^{2}+\beta^{2}}}\right),
\end{array}\right.
$$

are the parametrization of the ruled surfaces which are called Bertrandian Tangent ruled surface, Bertrandian Normal ruled surface and Bertrandian Binormal ruled surface, respectively [3].

\section{On the striction curves along the involutive and Bertrandian Darboux ruled surfaces based on the tangent vector fields}

In this section, first we give the twelve striction curves along twelve ruled surfaces which are called, collectively, Frenet ruled surface, Involutive Frenet ruled surface and Bertrandian Frenet ruled surface of curve $\alpha$. Further for each tangent 
vector fields of striction curves along Darboux, Involutive and Bertrand Darboux ruled surfaces have been calculated and orthogonality conditions are examined.

Definition 1. Let $\alpha^{*}(s)$ be involute of $\alpha(s)$ curve. The equation

$$
\varphi_{4}^{*}\left(s, v_{4}\right)=\alpha(s)+(c-s) V_{1}(s)+v_{4}\left(\frac{k_{2}}{\sqrt{k_{1}^{2}+k_{2}^{2}}} V_{1}-\frac{k_{1}^{\prime} k_{2}-k_{1} k_{2}^{\prime}}{\left(k_{1}^{2}+k_{2}^{2}\right)^{\frac{3}{2}}} V_{2}+\frac{k_{1} V_{3}}{\sqrt{k_{1}^{2}+k_{2}^{2}}}\right)
$$

is the parametrization of the ruled surface which is called involutive Darboux ruled surface.

Definition 2. Let $\left(\alpha, \alpha^{* *}\right)$ be Bertrand curves pair. The equation

$$
\varphi_{4}^{* *}\left(s, w_{4}\right)=\alpha+\lambda V_{2}+w_{4} \frac{\sqrt{\lambda^{2}+\beta^{2}}}{\left(\beta k_{1}-\lambda k_{2}\right)}\left(k_{2} V_{1}+k_{1} V_{3}\right)
$$

is the parametrization of the ruled surface which is called Bertrandian Darboux ruled surface.

Theorem 3. The striction curves $c_{1}, c_{2}, c_{3}, c_{4}$ of four Frenet ruled surfaces belonging to $\alpha$ curve is given by the following matrix

$$
\left[\begin{array}{c}
c_{1}-\alpha \\
c_{2}-\alpha \\
c_{3}-\alpha \\
c_{4}-\alpha
\end{array}\right]=\left[\begin{array}{ccc}
0 & 0 & 0 \\
0 & \frac{k_{1}}{k_{2}^{2}+k_{2}^{2}} & 0 \\
0 & 0 & 0 \\
\frac{-k_{2}}{k_{1}\left(\frac{k_{2}}{k_{1}}\right)} & 0 & \frac{-1}{\left(\frac{k_{2}}{k_{1}}\right)}
\end{array}\right]\left[\begin{array}{l}
V_{1} \\
V_{2} \\
V_{3}
\end{array}\right],[3]
$$

Theorem 4. Tangent vector fields $T_{1}, T_{2}, T_{3}$, and $T_{4}$ of striction curves along Frenet ruled surface are given by

$$
\left[\begin{array}{c}
T_{1} \\
T_{2} \\
T_{3} \\
T_{4}
\end{array}\right]=\left[\begin{array}{ccc}
1 & 0 & 0 \\
\frac{k_{2}^{2}}{\eta\left\|c_{2}^{\prime}(s)\right\|} & \frac{\left(\frac{k_{1}}{\eta}\right)^{\prime}}{\left\|c_{2}^{\prime}(s)\right\|} & \frac{k_{1} k_{2}}{\eta\left\|c_{2}^{\prime}(s)\right\|} \\
1 & 0 & 0 \\
\frac{\mu-\mu^{\prime}-\frac{k_{2}}{k_{1}}}{\mu\left\|c_{4}^{\prime}(s)\right\|} & 0 & \frac{\mu^{\prime}}{\mu^{2}\left\|c_{4}^{\prime}(s)\right\|}
\end{array}\right]\left[\begin{array}{l}
V_{1} \\
V_{2} \\
V_{3}
\end{array}\right]
$$

where $k_{1}^{2}+k_{2}^{2}=\eta,\left(\frac{k_{2}}{k_{1}}\right)^{\prime}=\mu$

Proof. It is easy to give this matrix, since we have already get the following equalyties,

$$
T_{1}(s)=T_{3}(s)=\alpha^{\prime}(s)=V_{1}
$$

Since $c_{2}(s)=\alpha(s)+\frac{k_{1}}{k_{1}^{2}+k_{2}^{2}} V_{2}$ and

$$
T_{2}(s)=\frac{c_{2}^{\prime}(s)}{\left\|c_{2}^{\prime}(s)\right\|}=\frac{k_{2}^{2}}{\left(k_{1}^{2}+k_{2}^{2}\right)\left\|c_{2}^{\prime}(s)\right\|} V_{1}+\frac{\left(\frac{k_{1}}{\eta}\right)^{\prime}}{\left(k_{1}^{2}+k_{2}^{2}\right)\left\|c_{2}^{\prime}(s)\right\|} V_{2}+\frac{k_{1} k_{2}}{\left(k_{1}^{2}+k_{2}^{2}\right)\left\|c_{2}^{\prime}(s)\right\|} V_{3}
$$


Also

$$
\begin{aligned}
& T_{4}(s)=\frac{c_{4}^{\prime}(s)}{\left\|c_{4}^{\prime}(s)\right\|}=\frac{\left(\left(\frac{k_{2}}{k_{1}}\right)^{\prime}\right)^{2}-\left(\frac{k_{2}}{k_{1}}\right)^{\prime}\left(\frac{k_{2}}{k_{1}}\right)^{\prime \prime}-\frac{k_{2}}{k_{1}}\left(\frac{k_{2}}{k_{1}}\right)^{\prime}}{\left(\left(\frac{k_{2}}{k_{1}}\right)^{\prime}\right)^{2}\left\|c_{4}^{\prime}(s)\right\|} V_{1}-\frac{-1\left(\frac{k_{2}}{k_{1}}\right)^{\prime \prime}}{\left(\left(\frac{k_{2}}{k_{1}}\right)^{\prime}\right)^{2}\left\|c_{4}^{\prime}(s)\right\|} V_{3}, \\
& T_{4}(s)=\frac{\mu^{2}-\mu \mu^{\prime}-\frac{k_{2}}{k_{1}} \mu}{\mu^{2}\left\|c_{4}^{\prime}(s)\right\|} V_{1}+\frac{\mu^{\prime}}{\mu^{2}\left\|c_{4}^{\prime}(s)\right\|} V_{3} .
\end{aligned}
$$

Theorem 5. The striction curves $c_{1}^{*}, c_{2}^{*}, c_{3}^{*}, c_{4}^{*}$ along four involutive Frenet ruled surfaces of curve $\alpha$ is given by the following matrix, in terms of Frenet apparatus of evolute curve $\alpha$, [3].

$$
\left[\begin{array}{c}
c_{1}^{*}-\alpha \\
c_{2}^{*}-\alpha \\
c_{3}^{*}-\alpha \\
c_{4}^{*}-\alpha
\end{array}\right]=\left[\begin{array}{ccc}
(c-s) & 0 & 0 \\
(c-s)\left(1-\frac{k_{1}^{2}}{\left(k_{1}^{2}+k_{2}^{2}\right)(1+m)}\right) & 0 & (c-s) \frac{k_{1} k_{2}}{\left(k_{1}^{2}+k_{2}^{2}\right)(1+m)} \\
(c-s) & 0 & 0 \\
(c-s)-\frac{k_{2}}{m^{\prime}\left(k_{1}^{2}+k_{2}^{2}\right)^{\frac{1}{2}}} & -\frac{m}{m^{\prime}} & \frac{k_{1}}{m^{\prime}\left(k_{1}^{2}+k_{2}^{2}\right)^{\frac{1}{2}}}
\end{array}\right]\left[\begin{array}{l}
V_{1} \\
V_{2} \\
V_{3}
\end{array}\right]
$$

Tangent vector fields of three striction curves along Darboux, Involutive and Bertrand Darboux ruled surfaces have been calculated.

Theorem 6. Tangent vector fields $T_{1}{ }^{*}, T_{2}{ }^{*}, T_{3}{ }^{*}, T_{4}{ }^{*}$ of striction curves of involutive Frenet ruled surface in terms of Frenet apparatus by themselves are given by

$$
\left[\begin{array}{c}
T_{1}{ }^{*} \\
T_{2}{ }^{*} \\
T_{3}{ }^{*} \\
T_{4}{ }^{*}
\end{array}\right]=\left[\begin{array}{ccc}
0 & 1 & 0 \\
\frac{-b^{*} k_{1}+c^{*} k_{2}}{\left(k_{1}^{2}+k_{2}^{2}\right)^{\frac{1}{2}}} & a^{*} & \frac{b^{*} k_{2}+c^{*} k_{1}}{\left(k_{1}^{2}+k_{2}^{2}\right)^{\frac{1}{2}}} \\
0 & 1 & 0 \\
\frac{e^{*} k_{2}}{\left(k_{1}^{2}+k_{2}^{2}\right)^{\frac{1}{2}}} & d^{*} & \frac{e^{*} k_{1}}{\left(k_{1}^{2}+k_{2}^{2}\right)^{\frac{1}{2}}}
\end{array}\right]\left[\begin{array}{l}
V_{1} \\
V_{2} \\
V_{3}
\end{array}\right]
$$

where

$$
a^{*}=\frac{k_{2}^{* 2}}{\eta^{*}\left\|c_{2}^{* \prime}(s)\right\|}, \quad b^{*}=\frac{\left(\frac{k_{1}^{*}}{\eta^{*}}\right)^{\prime}}{\left\|c_{2}^{* \prime}(s)\right\|}, c^{*}=\frac{k_{1}^{*} k_{2}^{*}}{\eta^{*}\left\|c_{2}^{* \prime}(s)\right\|}, \quad d^{*}=\frac{\mu^{*}-\mu^{* \prime}-\frac{k_{2}^{*}}{k_{1}^{*}}}{\mu^{*}\left\|c_{4}^{* \prime}(s)\right\|}, e^{*}=\frac{\mu^{* \prime}}{\mu^{* 2}\left\|c_{4}^{* \prime}(s)\right\|}
$$

and $k_{1}^{* 2}+k_{2}^{* 2}=\eta^{*},\left(\frac{k_{2}^{*}}{k_{1}^{*}}\right)^{\prime}=\mu^{*}$

Proof. From the equation (18), tangent vector fields $T_{1}{ }^{*}, T_{2}{ }^{*}, T_{3}{ }^{*}, T_{4}{ }^{*}$ of striction curves of involutive Frenet ruled surface matrix form as follows;

$$
\left[\begin{array}{c}
T_{1}^{*} \\
T_{2}^{*} \\
T_{3}^{*} \\
T_{4}^{*}
\end{array}\right]=\left[\begin{array}{ccc}
1 & 0 & 0 \\
a^{*} & b^{*} & c^{*} \\
1 & 0 & 0 \\
d^{*} & 0 & e^{*}
\end{array}\right]\left[\begin{array}{c}
V_{1}^{*} \\
V_{2}^{*} \\
V_{3}^{*}
\end{array}\right]
$$


In the above matrix by using the equation (3), we can write

$$
\left[\begin{array}{c}
T_{1}^{*} \\
T_{2}^{*} \\
T_{3}^{*} \\
T_{4}^{*}
\end{array}\right]=\left[\begin{array}{ccc}
1 & 0 & 0 \\
a^{*} & b^{*} & c^{*} \\
1 & 0 & 0 \\
d^{*} & 0 & e^{*}
\end{array}\right]\left[\begin{array}{ccc}
0 & 1 & 0 \\
\frac{-k_{1}}{\left(k_{1}^{2}+k_{2}^{2}\right)^{\frac{1}{2}}} & 0 & \frac{k_{2}}{\left(k_{2}^{2}+k_{2}^{2}\right)^{\frac{1}{2}}} \\
\frac{\left(k_{1}^{2}+k_{2}^{2}\right)^{\frac{1}{2}}}{k_{1}} & \frac{k_{1}}{\left(k_{1}^{2}+k_{2}^{2}\right)^{\frac{1}{2}}}
\end{array}\right]\left[\begin{array}{l}
V_{1} \\
V_{2} \\
V_{3}
\end{array}\right]
$$

or

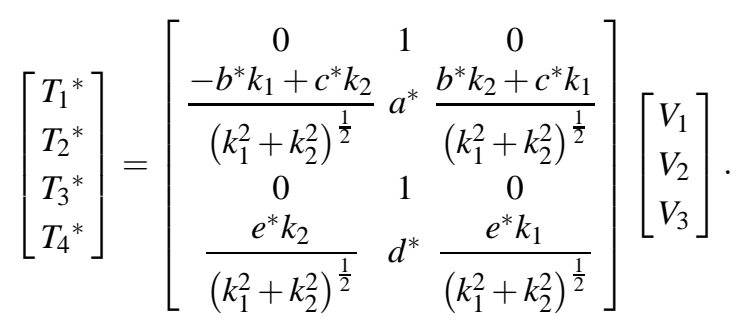

Now we can write striction curves along the "Bertrandian Frenet ruled surfaces" as in the following way in terms of the Frenet apparatus of curve $\alpha$.

Theorem 7. The striction curves $c_{1}^{* *}, c_{2}^{* *}, c_{3}^{* *}, c_{4}^{* *}$ along four Bertrandian Frenet ruled surfaces of curve $\alpha$ is given by the following matrix, in terms of Frenet apparatus of evolute curve $\alpha$, [3]

$$
\left[\begin{array}{c}
c_{1}^{* *}-\alpha \\
c_{2}^{* *}-\alpha \\
c_{3}^{* *}-\alpha \\
c_{4}^{* *}-\alpha
\end{array}\right]=\left[\begin{array}{ccc}
0 & \lambda & 0 \\
0 & \left(\lambda+\frac{m\left(\lambda^{2}+\beta^{2}\right) k_{2}}{\left(m^{2}+1\right)}\right) & 0 \\
0 & \lambda & 0 \\
\frac{m^{\prime} \sqrt{\lambda^{2}+\beta^{2}} k_{2}}{m^{3}} & \lambda & \frac{m^{\prime} \sqrt{\lambda^{2}+\beta^{2}} k_{1}}{m^{3}}
\end{array}\right]\left[\begin{array}{l}
V_{1} \\
V_{2} \\
V_{3}
\end{array}\right]
$$

Also using Frenet apparatus of involute curve $\alpha$, we can write excilipt equations of the striction curves of four Bertrandian Frenet ruled surfaces along the evolute curve $\alpha$ in terms of Frenet apparatus of evolute curve $\alpha$.

Theorem 8.Tangent vector fields $T_{1}^{* *}, T_{2}^{* *}, T_{3}^{* *}$, and $T_{4}^{* *}$ of striction curves of Frenet ruled surface are given by

$$
\left[\begin{array}{c}
T_{1}^{* *} \\
T_{2}^{* *} \\
T_{3}^{* *} \\
T_{4}^{* *}
\end{array}\right]=\left[\begin{array}{ccc}
\frac{\beta}{\sqrt{\lambda^{2}+\beta^{2}}} & 0 & \frac{\lambda}{\sqrt{\lambda^{2}+\beta^{2}}} \\
\frac{a^{* *} \beta-c^{* *} \lambda}{\sqrt{\lambda^{2}+\beta^{2}}} & b^{* *} & \frac{a^{* *} \lambda+b^{* *} \beta}{\sqrt{\lambda^{2}+\beta^{2}}} \\
\frac{\beta}{\sqrt{\lambda^{2}+\beta^{2}}} & 0 & \frac{\lambda}{\sqrt{\lambda^{2}+\beta^{2}}} \\
\frac{d^{* *} \beta-e^{* *} \lambda}{\sqrt{\lambda^{2}+\beta^{2}}} & 0 & \frac{d^{* *} \lambda+e^{* *} \beta}{\sqrt{\lambda^{2}+\beta^{2}}}
\end{array}\right]\left[\begin{array}{l}
V_{1} \\
V_{2} \\
V_{3}
\end{array}\right]
$$


where

$$
\begin{aligned}
& a^{* *}=\frac{k_{2}^{* * 2}}{\eta^{* *}\left\|c_{2}^{* * \prime}(s)\right\|}, b^{* *}=\frac{\left(\frac{k_{*}^{* *}}{\eta^{* *}}\right)^{\prime}}{\left\|c_{2}^{* *}(s)\right\|}, c^{* *}=\frac{k_{1}^{* *} k_{2}^{* *}}{\eta^{* *}\left\|c_{2}^{* *}(s)\right\|} \\
& d^{* *}=\frac{\mu^{* *}-\mu^{* * \prime}-\frac{k^{* *}}{k_{1}^{* *}}}{\mu^{* *}\left\|c_{4}^{* * \prime}(s)\right\|}=\frac{-m^{\prime}-\left(\frac{-m^{\prime}}{m^{2} k_{2} \sqrt{\lambda^{2}+\beta^{2}}}\right)^{\prime 2}-m k_{2} \sqrt{\lambda^{2}+\beta^{2}}}{-m^{\prime}\left\|c_{4}^{* * \prime}(s)\right\|} \\
& e^{* *}=\frac{\mu^{* * \prime}}{\mu^{* * 2}\left\|c_{4}^{* * \prime}(s)\right\|}=\frac{\left(\frac{-m^{\prime}}{m^{2} k_{2} \sqrt{\lambda^{2}+\beta^{2}}}\right)^{\prime} \frac{1}{k_{2} \sqrt{\lambda^{2}+\beta^{2}}}}{\left(\frac{-m^{\prime}}{m^{2} k_{2} \sqrt{\lambda^{2}+\beta^{2}}}\right)^{2}\left\|c_{4}^{* *}(s)\right\|}
\end{aligned}
$$

and $k_{1}^{* * 2}+k_{2}^{* * 2}=\eta^{* *},\left(\frac{k_{2}^{* *}}{k_{1}^{* *}}\right)^{\prime}=\mu^{* *}$

Proof. From the equation (20), tangent vector fields $T_{1}{ }^{*}, T_{2}{ }^{*}, T_{3}{ }^{*}, T_{4}{ }^{*}$ of striction curves of involutive Frenet ruled surface matrix form as follows;

$$
\left[\begin{array}{c}
T_{1}^{* *} \\
T_{2}^{* *} \\
T_{3}^{* *} \\
T_{4}^{* *}
\end{array}\right]=\left[\begin{array}{ccc}
1 & 0 & 0 \\
a^{* *} & b^{* *} & c^{* *} \\
1 & 0 & 0 \\
d^{* *} & 0 & e^{* *}
\end{array}\right]\left[\begin{array}{c}
V_{1}^{* *} \\
V_{2}^{* *} \\
V_{3}^{* *}
\end{array}\right]
$$

In the above matrix by using the equation (7), we can write

$$
\left[\begin{array}{c}
T_{1}^{* *} \\
T_{2}^{* *} \\
T_{3}^{* *} \\
T_{4}^{* *}
\end{array}\right]=\left[\begin{array}{ccc}
1 & 0 & 0 \\
a^{* *} & b^{* *} & c^{* *} \\
1 & 0 & 0 \\
d^{* *} & 0 & e^{* *}
\end{array}\right]\left[\begin{array}{ccc}
\frac{\beta}{\sqrt{\lambda^{2}+\beta^{2}}} & 0 & \frac{\lambda}{\sqrt{\lambda^{2}+\beta^{2}}} \\
0 & 1 & 0 \\
\frac{-\lambda}{\sqrt{\lambda^{2}+\beta^{2}}} & 0 & \frac{\beta}{\sqrt{\lambda^{2}+\beta^{2}}}
\end{array}\right]\left[\begin{array}{l}
V_{1} \\
V_{2} \\
V_{3}
\end{array}\right]
$$

or

$$
\left[\begin{array}{c}
T_{1}^{* *} \\
T_{2}^{* *} \\
T_{3}^{* *} \\
T_{4}^{* *}
\end{array}\right]=\left[\begin{array}{ccc}
\frac{\beta}{\sqrt{\lambda^{2}+\beta^{2}}} & 0 & \frac{\lambda}{\sqrt{\lambda^{2}+\beta^{2}}} \\
\frac{a^{* *} \beta-c^{* *} \lambda}{\sqrt{\lambda^{2}+\beta^{2}}} & b^{* *} & \frac{a^{* *} \lambda+b^{* *} \beta}{\sqrt{\lambda^{2}+\beta^{2}}} \\
\frac{\beta}{\sqrt{\lambda^{2}+\beta^{2}}} & 0 & \frac{\lambda}{\sqrt{\lambda^{2}+\beta^{2}}} \\
\frac{d^{* *} \beta-e^{* *} \lambda}{\sqrt{\lambda^{2}+\beta^{2}}} & 0 & \frac{d^{* *} \lambda+e^{* *} \beta}{\sqrt{\lambda^{2}+\beta^{2}}}
\end{array}\right]\left[\begin{array}{l}
V_{1} \\
V_{2} \\
V_{3}
\end{array}\right] .
$$

Theorem 9. Tangent vector fields of striction curves on Involutive tangent ruled surface and Bertrandian Darboux ruled surface have perpendicular for $\left\langle T_{1}^{*}, T_{4}^{* *}\right\rangle=0$. Tangent vector fields of striction curves on Involutive binormal ruled surface and Bertrandian Darboux ruled surface have perpendicular for $\left\langle T_{3}^{*}, T_{4}^{* *}\right\rangle=0$.

Proof. From the equations (19) and (21) the proof is completed.

Theorem 10. (i) Tangent vector fields of striction curves on Involutive Darboux and Bertrandian tangent ruled surfaces have perpendicular under the condition

$$
\left(\frac{-k_{2}^{2}\left(\frac{k_{1}}{k_{2}}\right)^{\prime}}{\left(k_{1}^{2}+k_{2}^{2}\right)^{\frac{3}{2}}}\right)^{\prime \prime}=0 .
$$


(ii) Tangent vector fields of striction curves on Involutive Darboux and Bertrandian binormal ruled surfaces have perpendicular under the condition

$$
\left(\frac{-k_{2}^{2}\left(\frac{k_{1}}{k_{2}}\right)^{\prime}}{\left(k_{1}^{2}+k_{2}^{2}\right)^{\frac{3}{2}}}\right)^{\prime \prime}=0 .
$$

Proof.

(i) From the equations (19) and (21), we get

$$
\begin{aligned}
\left\langle T_{4}^{*}, T_{1}^{* *}\right\rangle & =\left\langle\frac{e^{*} k_{2}}{\left(k_{1}^{2}+k_{2}^{2}\right)^{\frac{1}{2}}} V_{1}+d^{*} V_{2}+\frac{e^{*} k_{1}}{\left(k_{1}^{2}+k_{2}^{2}\right)^{\frac{1}{2}}} V_{3}, \frac{\beta}{\sqrt{\lambda^{2}+\beta^{2}}} V_{1}+\frac{\lambda}{\sqrt{\lambda^{2}+\beta^{2}}} V_{3}\right\rangle \\
& =\frac{e^{*}\left(k_{2} \beta+k_{1} \lambda\right)}{\left(k_{1}^{2}+k_{2}^{2}\right)^{\frac{1}{2}} \sqrt{\lambda^{2}+\beta^{2}}}=\frac{e^{*}}{\left(k_{1}^{2}+k_{2}^{2}\right)^{\frac{1}{2}} \sqrt{\lambda^{2}+\beta^{2}}} .
\end{aligned}
$$

Since $\left\langle T_{4}^{*}, T_{1}^{* *}\right\rangle=0$, we have

$$
\begin{aligned}
e^{*}=0 & \Longrightarrow \mu^{* \prime}=\left(\frac{k_{2}{ }^{*}}{k_{1}{ }^{*}}\right)^{\prime \prime} \frac{d s^{*}}{d s}=0 \\
& \Longrightarrow\left(\frac{-k_{2}^{2}\left(\frac{k_{1}}{k_{2}}\right)^{\prime}}{\left(k_{1}^{2}+k_{2}^{2}\right)^{\frac{3}{2}}}\right)^{\prime \prime} k_{1}(c-s)=0, k_{1} \neq 0 c=\text { const } . \\
& \Longrightarrow\left(\frac{-k_{2}^{2}\left(\frac{k_{1}}{k_{2}}\right)^{\prime}}{\left(k_{1}^{2}+k_{2}^{2}\right)^{\frac{3}{2}}}\right)^{\prime \prime}=0 .
\end{aligned}
$$

(ii) Since $\left\langle T_{4}^{*}, T_{1}^{* *}\right\rangle=\left\langle T_{4}^{*}, T_{3}^{* *}\right\rangle=e^{*}$, it is trivial.

Theorem 11. Tangent vector fields of striction curves on Involutive Darboux and Bertrandian Darboux ruled surfaces have perpendicular under the conditions

$$
e^{*}=0 \text { or } \frac{d^{* *}}{e^{* *}}=-\beta k_{1}+\lambda k_{2}
$$

Proof. From the equations (19) and (21), we get

$$
\begin{aligned}
\left\langle T_{4}^{*}, T_{4}^{* *}\right\rangle & =0 \Longrightarrow\left\langle\frac{e^{*} k_{2}}{\left(k_{1}^{2}+k_{2}^{2}\right)^{\frac{1}{2}}} V_{1}+d^{*} V_{2}+\frac{e^{*} k_{1}}{\left(k_{1}^{2}+k_{2}^{2}\right)^{\frac{1}{2}}} V_{3}, \frac{d^{* *} \beta-e^{* *} \lambda}{\sqrt{\lambda^{2}+\beta^{2}}} V_{1}+\frac{d^{* *} \lambda+e^{* *} \beta}{\sqrt{\lambda^{2}+\beta^{2}}} V_{3}\right\rangle \\
& =\frac{e^{*}\left(d^{* *}+e^{* *}\left(-\lambda k_{2}+\beta k_{1}\right)\right)}{\left(k_{1}^{2}+k_{2}^{2}\right)^{\frac{1}{2}} \sqrt{\lambda^{2}+\beta^{2}}}=0 .
\end{aligned}
$$

From this last equation, we can write

$$
e^{*}=0 \text { or } \frac{d^{* *}}{e^{* *}}=-\beta k_{1}+\lambda k_{2}
$$

Theorem 12. Tangent vector fields of striction curves on Involutive normal and Bertrandian Darboux ruled surfaces have perpendicular under the condition is

$$
\frac{b^{*} e^{* *}+c^{*} d^{* *}}{c^{*} e^{* *}-b^{*} d^{* *}}=\lambda k_{2}-\beta k_{1}
$$


Proof. From the equations (19) and (21), we get

$$
\begin{aligned}
\left\langle T_{2}^{*}, T_{4}^{* *}\right\rangle & =\left\langle\frac{-b^{*} k_{1}+c^{*} k_{2}}{\left(k_{1}^{2}+k_{2}^{2}\right)^{\frac{1}{2}}} V_{1}+a^{*} V_{2}+\frac{b^{*} k_{2}+c^{*} k_{1}}{\left(k_{1}^{2}+k_{2}^{2}\right)^{\frac{1}{2}}} V_{3}, \frac{d^{* *} \beta-e^{* *} \lambda}{\sqrt{\lambda^{2}+\beta^{2}}} V_{1}+\frac{d^{* *} \lambda+e^{* *} \beta}{\sqrt{\lambda^{2}+\beta^{2}}} V_{3}\right\rangle \\
& =\frac{b^{*} e^{* *}+c^{*} d^{* *}+\left(c^{*} e^{* *}-b^{*} d^{* *}\right)\left(-\lambda k_{2}+\beta k_{1}\right)}{\left(k_{1}^{2}+k_{2}^{2}\right)^{\frac{1}{2}} \sqrt{\lambda^{2}+\beta^{2}}} .
\end{aligned}
$$

Since $\left\langle T_{2}^{*}, T_{4}^{* *}\right\rangle=0$, we get

$$
\frac{b^{*} e^{* *}+c^{*} d^{* *}}{c^{*} e^{* *}-b^{*} d^{* *}}=\lambda k_{2}-\beta k_{1}
$$

Theorem 13. Tangent vector fields of striction curves on Involutive Darboux and Bertrandian normal ruled surfaces have perpendicular under the condition is

$$
\frac{e^{*}\left(a^{* *}+b^{* *} k_{1} \beta-c^{* *} k_{2} \lambda\right)}{d^{*} b^{* *}}=-\left(k_{1}^{2}+k_{2}^{2}\right)^{\frac{1}{2}} \sqrt{\lambda^{2}+\beta^{2}} .
$$

Proof. From the equations (19) and (21), we get

$$
\begin{aligned}
\left\langle T_{4}^{*}, T_{2}^{* *}\right\rangle & =\left\langle\frac{e^{*} k_{2}}{\left(k_{1}^{2}+k_{2}^{2}\right)^{\frac{1}{2}}} V_{1}+d^{*} V_{2}+\frac{e^{*} k_{1}}{\left(k_{1}^{2}+k_{2}^{2}\right)^{\frac{1}{2}}} V_{3}, \frac{a^{* *} \beta-c^{* *} \lambda}{\sqrt{\lambda^{2}+\beta^{2}}} V_{1}+b^{* *} V_{2}+\frac{a^{* *} \lambda+b^{* *} \beta}{\sqrt{\lambda^{2}+\beta^{2}}} V_{3}\right\rangle \\
& =\frac{e^{*} a^{* *}+e^{*}\left(b^{* *} k_{1} \beta-c^{* *} k_{2} \lambda\right)+d^{*} b^{* *}\left(k_{1}^{2}+k_{2}^{2}\right)^{\frac{1}{2}} \sqrt{\lambda^{2}+\beta^{2}}}{\left(k_{1}^{2}+k_{2}^{2}\right)^{\frac{1}{2}} \sqrt{\lambda^{2}+\beta^{2}}} .
\end{aligned}
$$

Since $\left\langle T_{4}^{*}, T_{2}^{* *}\right\rangle=0$, we get

$$
\frac{e^{*}\left(a^{* *}+b^{* *} k_{1} \beta-c^{* *} k_{2} \lambda\right)}{d^{*} b^{* *}}=-\left(k_{1}^{2}+k_{2}^{2}\right)^{\frac{1}{2}} \sqrt{\lambda^{2}+\beta^{2}} .
$$

\section{Competing Interests}

The authors declare that they have no competing interests.

\section{Authors' Contributions}

All authors have contributed to all parts of the article. All authors read and approved the final manuscript.

\section{References}

[1] Do Carmo, M. P., Differential Geometry of Curves and Surfaces, Prentice-Hall, ISBN 0-13-212589-7, 1976.

[2] Hacısalihoğlu H.H., Diferensiyel Geometri, Cilt 1, Ínönü Üniversitesi Yayinlari, Malatya, 1994.

[3] Kilıçoğlu Ş., Senyurt S. and Hacisalihoglu H. H. , On the Striction Curves of Involute and Bertrandian Frenet Ruled Surfaces in E3. Applied Mathematical Sciences, 9(142), 2015, 7081-7094, Doi: 10.12988/ams.2015.59606.

[4] Izumiya, S., Takeuchi, N., Special curves and Ruled surfaces, Beitrage zur Algebra und Geometrie Contributions to Algebra and Geometry,44(1), 203-212, 2003.

[5] Lipschutz M.M., Differential Geometry, Schaum's Outlines, 1969.

[6] Schief W., On the integrability of Bertrand curves and Razzaboni surfaces. Journal of Geometry and Physics, Volume 45, Issues 1-2, Pages 130-150, 2003. 